\title{
13 UBIQUITOUS COMPUTING AND THE DOUBLE IMMUTABILITY OF REMOTE DIAGNOSTICS TECHNOLOGY: An Exploration into Six Cases of Remote Diagnostics Technology Use
}

\author{
Katrin Jonsson \\ Jonny Holmström \\ Department of Informatics \\ Umeå University \\ Umeå, Sweden
}

\begin{abstract}
The aim of this paper is to display the use a specific type of ubiquitous computing technology — remote diagnostics technology—in organizations and, in particular, the way in which the technology is enacted in remote and local maintenance groups. By taking a case study approach, we look into the use of remote diagnostics technology in the maintenance industry. Drawing from actor-network theory, and in particular the notion of double immutability, we argue that we need to establish a stable relationship that uses remote diagnostics technology for monitoring machine performance from a remote place while also keeping a level of local responsiveness toward machine performance. The stability of the remote diagnostics technology is seemingly effective in that critical data can be collected, diffused, and manipulated. The stability of the network of relations surrounding the technology is, however, yet to emerge. The borders between the central group and the local maintenance workers must be considered and we need to acknowledge that it takes effort to sustain stable networks of relations. We need to establish a new relationship that uses ubiquitous computing technology for monitoring processes and activities from the remote group while also keeping a level of local responsiveness toward machine performance. Taken together, the remote and the local group, along with the remote diagnostics technology, constitute a maintenance work collective.
\end{abstract}




\section{INTRODUCTION}

Information technology has revolutionized contemporary business life as it has changed the ground rules of strategic management, marketing, logistics, and organizational design, to name but a few disciplines. As the most recent, and arguably most challenging, "wave of technology," ubiquitous computing technology is designed to blend into the surrounding and to serve specified purposes as knowledge work (Davis 2003), learning (Chae 2003), communication and collaboration (Grudin 2003) or businesses (Giles and Purao 2003; Medovich 2003). This wave of computerization is characterized by mobility and embeddedness (Avital and Germonprez 2003; Lyytinen and Yoo 2002) making it possible to collect large amounts of data from physical environments, users, and products. Ubiquitous computing is also characterized by a separation of data from the technology through which it was collected (Avital and Germonprez 2003) and the possibility to transfer it to remote places.

In this paper, we focus on a particular type of ubiquitous computing that is commonly referred to as remote diagnostics technology. Sensors and network access are installed into physical products, mostly machines, making it possible to collect and access their performance parameters remotely. The performance of products such as shipboard cranes and hydraulic engines can thereby be monitored in some detail to ensure continuous product performance and enable timely maintenance. Traditionally, monitoring is done periodically by local technicians visiting the product, but with remote diagnostics technology, central service centers can monitor products remotely. These remote groups can offer problem solving services as well as new value adding services, something that has been optimistically forecast for in the vehicle industry (Kuschel and Ljungberg 2004). To date, efforts in developing and using remote diagnostics technology have primarily been concerned with making maintenance work on the products more timely and effective.

The remote group enabled by remote technology is dependent on data collected by the system. The transformation of the machine's physical condition into a digital representation is crucial if remote diagnostics technology is to be adapted and used well. The remote group must rely on data displayed by the system, showing the details of the product's condition. Traditional local groups, on the other hand, are skilled in using their senses and manual collection of data as sources in maintenance planning. Remote diagnostics technology seems to challenge the way traditional local groups perform maintenance planning by enabling remote groups specialized in collecting and analyzing data.

The aim of this paper is to display the use of remote diagnostics technology in organizations, in particular the way in which the technology is enacted in central and local maintenance groups. There is a gap between those two groups that needs to be bridged in order to establish a maintenance collective that works well. Remote diagnostics technologies create a remote closeness between physically dispersed objects and actors, while at the same time creating a local physical distance when actors and objects move their interaction into the digital environment. Drawing from actor-network theory, and in particular the notion of ciouble immutability, we argue that we need to establish a stable relationship that uses remote diagnostics technology for monitoring machine performance from a remote place while also keeping a level of local responsiveness toward machine performance. Taken together, the remote and the local group, along with the remote diagnostics technology, constitute a maintenance work collective. 


\section{LITERATURE REVIEW}

\subsection{Ubiquitous Computing}

The development of information technology with increased computing power, smaller devices, and improved network capabilities has radically affected how and where computing technology can be used. The computer has moved from dedicated operating rooms, via the desktop into our homes, and has now reached traditional objects in our environment. Terms like traditional business computing, mobile computing, pervasive computing, and ubiquitous computing are used to describe this progress of technology and its usage (Lyytinen and Yoo 2002). The relation between those stages is outlined in Figure 1.

A low level of both embeddedness and mobility characterizes traditional business computing since the size of the computer makes it visible as well as stationary in the office environment. With decreased component size, the computer becomes mobile, making it possible to carry it with us. Input to the computer is still manual, so the amount of data is finite. When the level of embeddedness increases, the computer is provided with the capability to collect data automatically from the environment in which it is embedded. Pervasive computing is such an area where sensors, identifiers, and virtual models of the physical environment are used to provide the computer with information about the environment. The level of mobility is what differentiates pervasive computing from ubiquitous computing, the fourth type of computing mobility (Lyytinen and Yoo 2002).

Remote diagnostics technology is embedded into physical products, making them literally invisible. Sensors are installed to collect data from the product; the data are then transferred via network connections for further analysis. The level of mobility, that is the other characteristic of ubiquitous computing, could be interpreted in different ways. One way is to ask if the product is mobile or not. The answer to that question varies between the different cases in this study. Some products are mobile and have wireless network connections, but some products are stationary and transfer data via a permanent telecommunications network. Mobility could, however, be viewed from a user perspective and not from an infrastructure perspective. Remote diagnostics technology is designed and developed to enable seamless monitoring of the product's condition. Alarms are automatically sent to the responsible technician via e-mail or SMS. The technology thus enables mobile monitoring of the product, independent of whether or not the local technician is in the building. We interpret mobility in the perspective of the use the technology enables and not solely in the perspective of the infrastructure: that is, whether a wireless connection is required or not. Remote diagnostics technology is thus interpreted as a ubiquitous technology, both embedded and mobile.

The development of objects that are provided with tags and sensors allows interaction and automatic data collection. Passive and active tags, microprocessors, sensors, and transmitters have enabled a continuous collection and processing of data that is cheaper and less time consuming compared to manual interaction (Alt and Zimmermann 2001). Figure 2 illustrates how the physical world and the information world merge through the development and use of ubiquitous computing. 


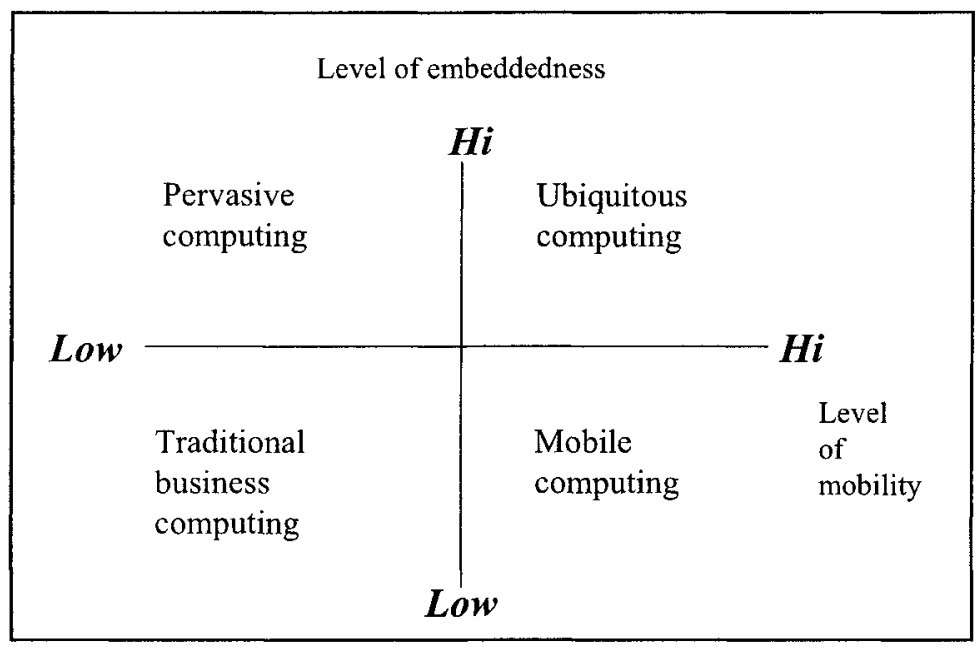

Figure 1. Different Types of Computing (from K. Lyytinen and Y. Yoo, "Issues and Challenges in Ubiquitous Computing," Communications of the $A C M(45: 12), 2002$, pp. 63-65. Used with permission).

\section{World of information (Bits, digital world)}

Internal and external information systems

Local, regional or global communication networks

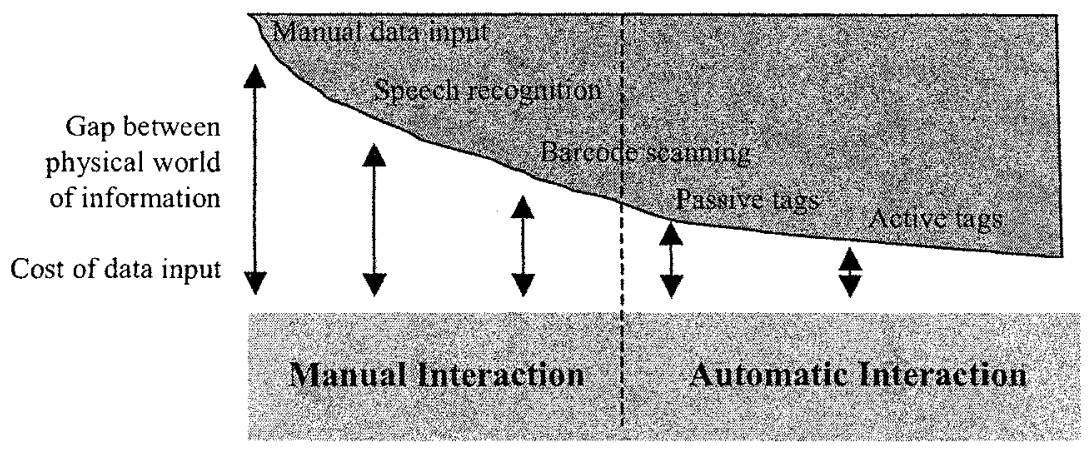

Physical world (Atoms)

Human beings, operating resources, products

Figure 2. The Merge of the Physical World and the Information World through Ubiquitous Computing (from E. Fleisch, "Von der Vernetzung von Undernehmen zur Vernetzung von Dingen," in Roadmap to E-Business, EM. Schögel, T. Tomczak, and Ch. Belz (Eds.), Verlag Thexis, 2002, pp. 124-136. Used with permission.) 
With traditional manual data input, the gap between the two worlds is high since the information processing is time consuming and noncontinuous, something that is changed with automatic data collection. Automatic interaction reflects the embedded perspective of ubiquitous computing as presented by Lyytinen and Yoo (2002) and the world of information enables mobility, which was the other characteristic they presented. The bits can flow in the digital network, making it possible for people to be mobile and be in different places and still have information access. In the case of remote diagnostics technology, the local group has developed skills based on the physical world, while the remote group is specialized in analyzing data in the digital world.

\subsection{Actor-Network Theory and Double Immutability}

With one group based on data analysis and another group skilled in physical assessments, a gap emerges. Drawing from actor network theory, and in particular the notion of double immutability, we argue that we need to establish a stable relationship that uses remote diagnostics technology for monitoring machine performance from a remote place while also keeping a level of local responsiveness toward machine performance.

The digital representation of a machine's condition is created through a transformation from the physical to the digital. A transformation process raises questions of what is gained and what is lost (Latour 2000). In Latour's studies of the transformation between the local and the universal, he showed how locality and particularity are lost in the transformation into the universal, while compatibility, standardization, and universality are gained. The transformation between the local and the universal described by Latour (2000) can be compared with the transformation between the physical and the digital with remote diagnostics technology. Both transformation processes raise questions of what is gained and what is lost and they both deal with the local and the remote. The digital world could be compared with the universal, and the physical world could be compared with the local. In order to succeed with the transformation between the local and the universal the object must have the properties of being mobile but also presentable, readable and combinable with one another, labeled by Latour (1987) as immutable mobility. The immutable mobile can move in time and space more or less unchanged. The immutable mobile (Law 1987; Mol and Law 1994; Singleton and Michael 1993) could, for example, be writings, maps, graphs, figures, and formulas.

Data in the digital world could be described as immutable mobile in the sense that it can be transferred all over the world and still be the same. Networks make it perfectly mobile by making it possible to transfer data thousands of kilometers in milliseconds. It is also possible to combine binary data. We can receive files from different sources, open them in the same computer, cut and paste them into the same file, and then send it further. The immutable mobile makes it possible for actors on the periphery to become familiar with events that are remote by giving them access to stable information; they can act at a distance (Latour 1987).

An immutable mobile provides a way of structuring vision and, moreover, enabling whatever is presented to be rapidly manipulated and controlled and it can be understood 
as the product of a series of transformations where inscriptions and translation processes are played out. Put into a technical context, inscription refers to the way technical artefacts embody patterns of use. As programs of actions are inscribed into technology, it becomes an actor that imposes its inscribed program of action on its users (Hanseth and Monteiro 1998). Inscriptions may vary in strength. Strong inscriptions leave little room for interpretative flexibility, whereas weak inscriptions leave some room for interpretation (Monteiro 2000). Translation refers to the process in which actors reinterpret and appropriate each other's interest to their own. Translation also refers to the process in which the meaning of the technology is negotiated with other elements in the environment. This process of translation embeds technology into the context of use, producing stability and social order (Callon 1986; Mähring et al. 2004).

Immutable mobiles are, in effect, examples of strong inscriptions. They are immutable in that they move in time and space more or less unchanged. But we need to pay close attention to the double nature of immutability.

Thus the first take-home lesson from ANT and post-ANT studies is that objects often display-may be understood as being constituted in-a double immutability. On the one hand they probably have a more or less stable shape in physical space - though the definition of that stable physical shape is likely to depend on relational and interactive work of one kind or another (and it may also be that "abstract objects" don't occupy Euclidean space). On the other, they certainly have, display, or are constituted by, a more or less stable structure in a network of relations (Law and Singleton 2003, p. 6).

This notion of double immutability leads us to focus not only on remote diagnostics technology per se, but also on the network of relations in which it is embedded and the processes of translation of which it is part. In exploring this, following Law and Singleton (2003), we should pay close attention to what they refer to as the "double immutability": the stability of entities both in their physical shape and in their network of relations.

\section{TRAVELING BETWEEN THE REMOTE AND THE LOCAL IN THE CONTEXT OF MAINTENANCE WORK}

\subsection{Method}

This article is based on qualitative case studies of companies developing or using remote diagnostics technology within the maintenance industry. Six different organizations with different relations to this kind of systems have been part of the study. The organizations are MacGregor Cranes, Monitoring Control Centre (MCC), Alpha, Beta, Gamma, and PowerDrive (fictitious names on the last four). MacGregor is a manufacturer of cranes and has developed a remote diagnostics system. MCC is a service provider that offers different services based on remote diagnostics. Alpha and Beta are 
organizations that use remote monitoring/diagnostics in their plants. Gamma and PowerDrive are both manufacturers that have developed their own systems and offer services based on these. All companies thus have experience of either developing or using this kind of system, which give us a more overarching view of the area of remote diagnostics and the gains and losses with this kind of system from a local and remote perspective. In total, three organizations are manufacturers developing remote diagnostics technology. One organization is a pure service provider that bases their services on collected data. Two organizations are customers who have systems installed in their plants. In total, 31 people have been interviewed, ranging from engineers, technicians, after-sales personnel, remote diagnostics developers, maintenance managers, and sellers. All interviews have been recorded and transcribed. The interviews have ranged from 45 minutes to 3 hours and have been focused on how this kind of system is used and what experiences the person has from working with the technology.

Our interviews tapped into the experiences of working with remote diagnostics technology, with a particular emphasis on maintenance work. One of the aims with remote diagnostics is to enhance the maintenance process by enabling predictive or condition-based maintenance. The technology is used for monitoring and the maintenance decisions are based on both scheduled and actual condition of the product (Campbell 1995). In the following section, we will describe the use of remote diagnostics technology from a local and a remote perspective.

\subsection{Remote Diagnostics Technology: A Remote Perspective}

When a digital model of a physical world is created (the world of information), it becomes possible to do calculations on collected data. When the world of information is growing, the computing model contains more and more data about the product's condition and analyses are done in order to detect abnormal conditions and trends. In the maintenance industry, there seems to be a trend toward collecting more and more data to get an even more all-embracing model of the physical object. The director of maintenance at Alpha expresses it as,

\section{We do measure parameters that we don't get any benefit of. But if you haven't measured them, the data is lost.}

When this world of information is growing, it creates new conditions for how the condition-based maintenance could be performed. Remote monitoring of products makes it possible to collect data, analyze it, and do predictions at a distance from the physical product. Since the data gathering is automatic and the data could be transferred via a network, the physical dependency disappears. MCC, for example, has one office in the northern part of Sweden but envisions becoming a global service provider without opening any new offices. MacGregor is another company highlighting decreased physical dependency in promotion of their remote diagnostic service. In their brochures, their service is promoted as, 
We provide seamless service solutions....we monitor the condition and performance of your cargo handling equipment, wherever the ship is in the world.

The world of information can be created without an engineer physically visiting every product. The development of automated data collection with a decreased gap between the physical and the digital world allows for a new kind of condition-based monitoring. The engineers no longer have to visit the products; instead, more of their daily work is done in the office and consists of analyzing collected data. This data lets the engineer detect abnormal variations and, hopefully, prevents breakdowns. To companies offering services based on collection and analysis of data, the technology allows for a seamless service solution since the technology is always present in the plant.

Automated data collection also creates a stable and regular flow of data, whereas manual collection could result in corrupt data due to irregularities in time and use of instruments. A developer of remote diagnostics systems at Gamma view it as,

Manual measuring is not acceptable. People are not good at repeatable accuracy. You don't pressure [the instrument] in the same way each time, you don't hold it in the same way and you are not able to place the measuring circuit in the same position each time. What people are good on are analyses.

With computerized measuring, the regularity and measuring technique is always the same. Computerized automated data collection will thus enhance the creation of a stable and regular data collection. The Gamma developer indicated that this would make the data automatically collected more reliable. The stability of data would thus be enhanced.

The technical development has also allowed for other actors to offer services based on remote monitoring of products. A manufacturer can collect data from a product located in another part of the world, analyze it, and take actions based on the information. PowerDrive has developed a remote service offer where they collect data from products all around the world and then transfer it back to their main office where analysis takes place. Collection of data from many dispersed objects in one place enhances the possibility of comparing data and the people doing the analyses get experience from many different machines and settings. MCC and MacGregor have developed similar services. MacGregor promotes their service as, "Wherever you arewe are there." This should, of course, be interpreted in a virtual sense. MacGregor is not present physically but their remote diagnostics system, through which they can follow the use and condition of the crane in its actual environment, is always present. MacGregor is thus present in the crane's environment in a virtual manner via the technology.

To the manufacturer, the use of remote diagnostics technology is an interesting area since it creates new business opportunities in the after-sale market while at the same time giving them important feedback about the products in use. With remote monitoring of products the manufacturer gets a picture of how they work in actual settings and if common problems within product runs exist. PowerDrive, for example, has been using their system to test products before they are launched to the market. To PowerDrive, the 
system has been valuable in product development since it gives them an overall picture about certain product categories.

\subsection{Remote Diagnostics Technology: A Local Perspective}

Although the products are constantly monitored with remote diagnostics technology, only a specified number of parameters can be collected. Thus, the picture the distant technician gets is not total. As the director at MCC expresses it,

When you physically walk around in the plant, you get a lot of other signals; you see, hear, and feel.

The diagnoses that can be performed with the system are limited to the number of parameters that are monitored. A physical walk-around by an experienced engineer who can see, hear, and feel when something is abnormal can detect things that the remote diagnostics technology cannot. As a hydraulic engineer at Beta expresses it,

You have to learn to know the machines individually. You don't do that remotely.

This highlights a clear limitation with the remote diagnostics technology compared to an experienced engineer walking around in the plant. Although the system can detect conditions by detailed analysis that the engineer cannot, many of the respondents point out the importance of keeping in mind that there is a world out there and the collected data on its own is not enough. The limitations of the technology are expressed by the hydraulic engineer at Beta as,

With more monitoring, you can work more and more online and less people will be out among the machines. You won't get the same feeling of the machines. The only things you see are, for example, temperature, pressure, and flow. You don't know how it sounds.

The system and, therefore, also the remote technicians are limited to the data collected by the sensors. The limited depiction of the reality is a technological constraint that always has to be kept in mind and considered. Another constraint related to this kind of system is highlighted in an event at Alpha. An engineer at the company described how they installed a diagnostics system at one of the oil pumps that monitored the oil level. This system was supposed to be a complement to the operators' traditional regular walk rounds in the plant. When the oil level sank, an engineer located in another building called the operator and instructed him to fill it with more oil. After a while the operator got used to the phone calls and stopped walking around in the plant. The system, however, only measured the oil level and did not give the engineer in the office an overall depiction of the machine and its environment. One day the machine almost broke down due to other problems that the system did not detect, but the operator would 
have seen it if he had walked by. To him, the phone calls had become a work practice indicating problems and, therefore, he viewed the walk rounds as unnecessary.

The example highlights the constraint of remote diagnostics technology described earlier; the depiction of the reality is limited and all problems cannot be detected. But the example also gives us insight into another potential constraint with the remote technology: the technology can create a remote closeness where engineers in another room, house, organization, or even part of the world can get close to the equipment. However, at the same time, this may create a local physical distance in the plant, as happened in the case above. The operator stopped walking by the machine physically since he relied on the digital information environment and the procedure of getting an alarm when something had to be done. The operator experienced that the use of the technology created new working procedures on which he comfortably relied. The example highlights the benefit of a remote closeness but, more important, it shows a potential loss when a local physical distance is created. The operator in this case stopped doing the walk rounds and, although the operator was in the same building as the machine, the technological environment made him view the traditional physical closeness as unnecessary; thus a local physical distance was created. As has been pointed out, the technology has its own benefits and the physical walks rounds by the local group have other benefits.

\subsection{The Relation Between the Local and the Remote Perspective}

Both the local and the remote group seem to have their own benefits and constraints. All of the organizations involved in this study report perceived benefits related to the use of remote diagnostics technology in terms of an increased remote awareness of their processes. There are advantages for maintenance work in terms of a better understanding of how machines and procedures function. The advantages to the local group are, however, also raised. Physical presence gives information impossible to transform into the digital world.

The introduction of remote diagnostics technology makes it important to handle the relationship between the two groups, especially since there seems to be a perceived shift in responsibility in the eyes of the local group. As the director at MCC expresses it,

It's very important that the border between the two groups is clear. The local groups must be well fuctioning...they must have basic control of the machines. This doesn't work today. The borders are very unclear. When the company buys services from the remote group, the local group seems to view the remote group as responsible for everything, at the same time as the remote group views the local group as responsible for basic monitoring and walk rounds. This happens when the borders are not clear; the responsibility has to be clearly expressed.

The increased "remote closeness" came as a result of the use of remote diagnostics technology, but we could also see a certain increase in the "local physical distance," 
which has to do with local groups assessing walk rounds as unnecessary and shifting responsibility. This seems to be a challenge to be bridged.

\section{DISCUSSION}

The findings illustrate how remote diagnostics technology is a ubiquitous and increasingly critical part of the fabric of the modern organization, supporting its operations. In particular, remote diagnostics technology influences which organizational actions, and their consequences, become more visible. Arguably more than anything else, ubiquitous computing is characterized by a separation of data from the technology through which it was collected (Avital and Germonprez 2003) and the collected data represents a digital model of the physical world. Introducing an actor-network perspective on these cases highlights the role that remote diagnostics technology plays in shaping organizational realities through the norms and values embedded within it. We focus on double immutability in this process, and, taken together, this gives us insight into the relationship between the central and the local maintenance groups in the maintenance of products embedded with remote diagnostics technology.

\subsection{The Local and the Remote}

The remote diagnostics technology has in these cases shown a degree of immutability in its character. Respondents at all companies view the technology as effective in that critical data can be easily collected, diffused, and analyzed. The data is perceived as stable in the sense that it can be transferred via the digital network to remote service groups without being corrupted. The involved companies see a great potential in the possibility of combining data from dispersed products, making it possible to compare them and hopefully drawing valuable conclusions. The three characters of the immutable mobile - mobility, stability, and combinability - thus seem to be characteristics suited for remote diagnostics technologies. The other side of double immutability concerns the stability of the network of relations. In our cases, it seems as if this stability is a challenge yet to be bridged.

The digital world of information enabled by remote diagnostics technology can be seen as an abstracted view of the machines in the physical world; it is a snapshot including some specified parameters. On an aggregate level, these parameters are to capture the performance of the machine in which the remote diagnostics technology is embedded. Focusing on the transformation between the local and the remote can help to explain the remote diagnostics process. As is told by some of the respondents, a physical visit to the machines give maintenance workers information through all of the senses. This is lost when the machine is diagnosed remotely via a specified number of parameters. The sensors only detect what they are designed to detect; the local maintenance worker can get additional information other than the parameters he his checking. One should, however, bear in mind that the sensors can register data that 
maintenance workers cannot, but nevertheless a sense of locality is lost when specified data is collected and transferred away via the digital network to a remote place.

While qualities related to locality are lost, factors like compatibility and relative universality are gained with remote diagnostics technology. The transformation of the machine's condition into digital numbers makes it possible for a central service center to compare different machines with their ideal condition and with each other, independent of their physical location. It also becomes easier to do calculations in order to predict what is about to happen with the machine in order to perform timely maintenance. The technology thus enables a remote closeness in the sense that the central service center can constantly diagnose the product, independent of the physical distance. They can, in a virtual sense, always be present in order to help the customers maintain their products. At the same time as the remote closeness is gained, there is a potential loss. As illustrated by the cases in this study, the remote closeness can create a local distance when the monitoring of the machine is digitalized. The remote group cannot collect the same data as the local technician, nor can the technician collect the same detailed data as the remote group do via the technology. When diagnostics are based solely on technology and the responsibility is shifted to the remote group a local physical distance emerges. The network of relations between those two groups must be clear and stable so each groups' benefits are used, otherwise the local distance may destroy the benefits of the central service group when remote diagnostics technology is to be used.

\subsection{Creating a Maintenance Collective}

Remote technology is used for leveraging the "digital information world"representation of machine performance of importance for maintenance work. This enable more effective control from the side of the central service group. In the companies studied, both the local maintenance group and the remote service group examined in this study were aware of both their own and the other party's roles and responsibilities. The remote group knew that they were not able to diagnose the product to 100 percent. The local group perceived their own work in the same way; they knew they were not able to predict the condition of the machines with the same precision as the remote diagnostics technology.

Although both parties were aware of the limitations in their own work, the network of relations between them has not been addressed in such a way that seems to be necessary in order to establish a maintenance collective that works well. Instead the local groups perceived a shifted responsibility from themselves to the central groups, which can have damaging effects on maintenance work. Neglected maintenance from the local group could result in an overall unsuccessful maintenance strategy since these two groups do not replace each other. Instead, they should be viewed as complementary and, woven together, they could constitute a strong maintenance collective.

Through such a collective, stability in the network of relations can be achieved. This other face of immutability thus seems to be something that the involved parties have to take into consideration during the design work. Instead of focusing only on the technology per se and the remote group's work, both faces of the immutability have to 
be considered. Thereby, a maintenance collective can emerge where all parties-the technology, the remote group, and the local group-contribute to the double immutability.

\section{CONCLUSIONS}

The purpose of this paper has been to display the use of remote diagnostics technology in organizations, in particular the way in which the technology is enacted in central and local maintenance groups. To address the various circumstances that surround the use of remote diagnostics technology, the notion of double immutability was introduced and discussed. This provided a language to talk about the efforts needed to establish a maintenance collective that works well and that can face the challenges involved in the maintenance of machines in which remote diagnostics technology has been embedded.

The results revealed that information technology was used for leveraging the information world-the representation of machine performance of importance for maintenance work. In our cases, we have seen how the remote diagnostics technology creates a remote closeness between objects and actors physically dispersed but at the same time it creates a local physical distance when actors and objects physically close to each other move their interaction into the ubiquitous computing environment. The possibilities to transfer data have opened up the possibilities to get knowledge about objects that are distant and combine data from these physically dispersed objects, which enhances the creation of an overall depiction. Ubiquitous computing could thus enhance the creation of a global depiction of remote phenomenon.

Following Law and Singleton (2003) and their idea of the double immutabilitythat the stability of entities is found both in their physical shape and in a network of relations-we find that stability is sustained in two partially related ways. The stability of the remote diagnostics technology is seemingly effective in that critical data can be collected, diffused, and manipulated. But the stability of the network of relations surrounding the technology is yet to emerge. In our application of the notion of double immutability, we identified the challenge of better integrating central and local maintenance group in the maintenance of products embedded with remote diagnostics technology. The borders between the remote group and the local maintenance workers must be considered and we need to acknowledge that it takes effort to sustain stable networks of relations. We need to establish a new relationship that uses ubiquitous computing technology for monitoring processes and activities from the central group while also keeping a level of local responsiveness toward machine performance. Taken together, the remote and the local group, along with the remote diagnostics technology, could constitute a maintenance work collective.

\section{REFERENCES}

Alt, R., and Zimmermann, H.-D. "Introduction to Special Section-Business Models," Electronic Market (11:1), 2001, pp. 3-9. 
Avital, M., and Germonprez, M. "Ubiquitous Computing: Surfing the Trend in a Balanced Act," paper presented at the Workshop on Ubiquitous Computing Environment, Cleveland, OH, October 23-26, 2003.

Callon, M. "Some Elements of a Sociology of Translation: Domestication of the Scallops and Fishermen of St Brieuc Bay," in Power, Action and Belief: A Sociology of Knowledge?, J. Law (Ed.), London: Routledge and Kegan Paul, 1986, pp. 196-233.

Campbell, J. D. Uptime: Strategies for Excellence in Maintenance Management, Portland, OR: Productivity Press, 1995.

Chae, B. "Ubiquitous Computing for Mundane Knowledge Management: Hopes, Challenges and Questions," paper presented at the Workshop on Ubiquitous Computing Environment, Cleveland, $\mathrm{OH}$, October 24-26, 2003.

Davis, G. B. "Affordances of Ubiquitous Computing and Productivity in Knowledge Work," paper presented at the Workshop on Ubiquitous Computing Environments, Cleveland, $\mathrm{OH}$, October 24-26, 2003.

Fleisch, E. "Von der Vernetzung von Unternehmen zur Vernetzung von Dingen," in Roadmap to E-Business, M. Schönegel, T. Tomczak, and Ch. Belz (Eds.), St. Gallen, Switzerland: Verlag Thexis, 2002, pp. 124-136.

Giles, C. L., and Purao, S. "The Role of Search in Ubiquitous Computing," paper presented at the Workshop on Ubiquitous Computing, Cleveland, OH, October 23-26, 2003.

Grudin, J. "Implications of Technology Use throughout Organizations," paper presented at the Workshop on Ubiquitous Computing, Cleveland, OH, October 23-26, 2003.

Hanseth, O., and Monteiro, E. "Changing Irreversible Networks: Institutionalization and Infrastructure," in Proceedings of $6^{\text {th }}$ European Conference on Information Systems, W. R. J. Baets (Ed.), Euro-Arab Management School, Granada, Spain, 1998, pp. 1123-1139.

Kuschel, J., and Ljungberg, F. "Decentralized Remote Diagnostics: A Study of Diagnostics in the Marine Industry," in People and Computers XVIII: Design for Life, S. Fincher, P. Markopoulos, D. Moore, and R. Ruddell (Eds.), London: Springer, 2004,pp. 211-226.

Latour, B. Pandora's Hope: Essays on the Reality of Science Studies, Cambridge, MA: Harvard University Press, 2000.

Latour, B. Science in Action, Cambridge, MA: Harvard University Press, 1987.

Law, J. "On the Methods of Long-Distance Control: Vessels, Navigation and the Portuguese Route to India," in Power, Action and Belief: A New Sociology of Knowledge, J. Law (Ed.), London: Routledge and Kegan Paul, 1987, pp, 234-263.

Law, J., and Singleton, V. Object Lessons, Lancaster, UK: The Centre for Science Studies, Lancaster University, 1987.

Lyytinen, $\mathrm{K}$., and Yoo, Y. "Issues and Challenges in Ubiquitous Computing," Communications of the $A C M(45: 12), 2003$, pp. 63-65.

Mähring, M., Holmström, J., Keil, M., and Montealegre, R. "Trojan Actor-Networks and Swift Translation: Bringing Actor-Network Theory to IT Project Escalation Studies," Information Technology and People (17:2), 2004, pp. 210-238.

Medovich, M. "Pervasive Computing and Pervasive Economies in the $21^{\text {st }}$ Century," paper presented at the Workshop on Ubiquitous Computing Environment, Cleveland, $\mathrm{OH}$, October 23-26, 2003.

Mol, A., and Law, J. "Regions, Networks and Fluids: Anaemia and Social Topology," Social Studies of Science (24:4), 1994, pp. 641-671.

Monteiro, E. "Actor-Network Theory," in From Control to Drift: The Dynamics of Corporate Information Infrastructure, C. U. Ciborra, K. Braa, A. Cordella, B. Dahlbom, A. Failla, O. Hanseth, V. Hespo, J. Ljungberg, E. Monteiro, and K. A. Simon (Eds.), Oxford: Oxford University Press, 2000, pp. 71-83.

Singleton, V., and Michael, M. "Actor-Networks and Ambivalence: General Practitioners in the UK Cervical Screening Programme,” Social Studies of Science (23:2), 1993, pp. 227-264. 


\begin{abstract}
ABOUT THE AUTHORS
Katrin Jonsson is a Ph.D. student and instructor at the department of Informatics, Umeå University, Sweden. Her research is situated within ubiquitous information environments and investigates its implications in organizations. Her thesis project focuses on remote diagnostics technology use in the industry and she is collaborating with a number of manufacturing and process-industry companies. Katrin can be reached at katrin.jonsson@informatik.umu.se.

Jonny Holmström is an assistant professor in Informatics at the University of Umeå, Sweden and a research manager the Centre of Digital Business. His current research includes empirical examinations of innovation networks with particular emphasis on the technologies that support them. He has published his research in Information and Organization, Information Technology and People, Journal of Global Information Technology Management, Scandinavian Journal of Information Systems, and at major international conferences. Jonny can be reached at jonny.holmstrom@informatik.umu.se
\end{abstract}

